\title{
In Terms of Analgesic Requirements - A Comparison Between Laparoscopic Nephrectomy and Open Nephrectomy
}

\author{
Abdul Matin Anamur Rashid Choudhury ${ }^{1}$, Tohid Mohammad Saiful Hossain ${ }^{2}$, Tasmina Parveen ${ }^{3}$, \\ Mohammed Mizanur Rahman ${ }^{4}$ Md. Safiul Alam Babul ${ }^{5}$
}

Received: 15 - 05 - 2020

Accepted: $29-07-2020$

Conflicts of interest: None

Keywords: Laparoscopic nephrectomy, open nephrectomy, pain, pethidine consumption.

\begin{abstract}
:
Objective: In this study, we aimed at making a comparison between the laparoscopic nephrectomy and open nephrectomy in terms of post-operative pain and pethidine consumption.
\end{abstract}

Methods: The study has been designed with patients who underwent prospective and randomized laparoscopic and open nephrectomy operation. We assessed the post-operative pain through visual analogue scale (VAS) at the 0st, 2nd, 4th, 8th, 12th, 18th, and 24th post-operative hours. We carefully recorded the intraoperative and post-operative results along with the post-operative pain-relieving analgesic doses.

Results: A total number of 30 patients were included in this study. The VAS scores of the two groups did not demonstrate a significant difference, but the analgesic (Pethidine) requirement was significantly lower in the laparoscopic group $(p=0.031)$. The groups demonstrated no difference in terms of adverse effects.

Conclusion: Along with similar perioperative results, laparoscopic nephrectomy offers an advantage in case of post-operative pain with regard to analgesic requirement and hospital stay duration.

\section{Introduction:}

Endourological interventions have replaced many classical surgical intervention methods. The most important reason for the preference of these surgical intervention methods by surgeons lies in their advantage of being minimally invasive. In this respect, ureterorenoscopic, percutaneous renoscopic and laparoscopic treatment approaches are the most preferred treatment methods in today's urology. However, classical surgery remains as the best alternative in some indications. In general, subcostal flank incision is used in open surgical approaches employed for upper urinary system. This approach offers the surgeon a wide operative field. However, according to the general consensus, it requires a considerable amount of muscle cutting, and leads to more post-operative pain and longer recovery period. Since it leads to pain, secretion of the potent mediators of inflammation, and tissue trauma, surgery might be considered as an injury. ${ }^{1}$ Consequently, post-operative pain may be present in an expected case, but it is not expected to be the same in all surgical procedures. ${ }^{2}$

1. Associate Professor, Department of Urology, National Institute of Kidney diseases and Urology (NIKDU), Dhaka

2. Associate Professor, Department of Urology, Bangabandhu Sheikh Mujib Medical University (BSMMU), Dhaka.

3. Professor, Department of Physiology, Uttara Adhunik Medical College, Dhaka

4. Associate Professor, Department of Urology, Patuakhali Medical College, Patuakhali

5. Assistant Professor \& RS, Department of Urology, National Institute of Kidney diseases and Urology (NIKDU), Dhaka

Correspondence: Dr.Abdul Matin Anamur Rashid Choudhury, Associate Professor, Department of Urology, National Institute of Kidney diseases and Urology (NIKDU), Dhaka, Bangladesh. E-mail: dr_choudhury2001@yahoo.com 
There is a close association between the effective management of post-operative pain and patient satisfaction, early mobilization, short hospital stays and decrease in costs. Therefore, routine surgical procedures shall principally consider reducing the post-operative pain.

\section{Methods:}

Inclusion criteria : Patients with an ASA physiological condition of I-III, who were planned for elective surgery early in the morning ( 8 am-3 pm), were included in the study.

\section{Exclusion criteria:}

Morbid obesity (BMI >35), central nervous system dysfunctions psychiatric diseases, substance-use, chronic or recent analgesic use ( $<2$ months), cardiovascular, hepatic or renal failure, pregnancy being underage ( $<18$ years).

All patients were prepared in the same way (informed consent form, bowel prep, etc.) in the preoperative process. The patients were operated with the same surgical technique, by the same experienced surgeons and the same anesthesia team. We standardized the intra-operative anesthesia protocol and applied for all cases. We monitored all patients through electrocardiogram, non-invasive blood pressure, endtidal $\mathrm{CO} 2$, and oxygen saturation. We used 1,5-2 mg/ $\mathrm{kg}$ propofol for the general anesthesia induction; rocuronium infusion for promoting intra-operative muscle relaxation; $2-3 \mu \mathrm{g} / \mathrm{kg}$ fentanyl for intraoperative analgesia; and a mixture of oxygen nitric oxide enriched with isoflurane (ratio 1:1) to the extent deemed necessary by the anesthesiologist for the inhalation anesthesia. Prior to the surgical procedure, we performed nasogastric tube and urinary catheter insertion for all patients. We positioned the patients into lateral lumbotomy position under general anesthesia, and the operation table, therefore the patient were fixed in a 30 degree oblique position toward the surgeon. We performed laparoscopic nephrectomy through the transperitoneal or retroperitoneal track in accordance with the principles previously defined in detail by the literature ${ }^{3}$. We performed the laparoscopic surgical procedure in lateral decubitus position with $12 \mathrm{mmHg}$ $\mathrm{CO} 2$ insufflation by employing laparoscopy standard automatic insufflator and we did not use local analgesic during the peroperative period.

We reversed the neuromuscular relaxation pharmacologically at the end of the operation by using atropine and neostigmine. We evaluated the postoperative pain by using the 10-point VAS taught the patients in the premedication period. In addition to the pain scores, we also recorded data regarding nausea, vomiting, dizziness, fatigue, loss of concentration, blurred vision, itching, number of post-operative vomits, as well as the amount of the analgesic used at the 0th, 2nd, 4th, 6th, 8th, 12th and 24th hours. All patients received single-dose prophylactic antibiotic 1 hour before the operation, and low-molecular-weight heparin and antithrombotic prophylaxis through a week starting one day before the operation. The patients wore compression stockings until the full-mobilization. Surgical operations like, advanced laparoscopic procedures and traditional open surgery were performed by the same experienced surgical team

Statistical analysis: In order to compare the classifiable variables, we used the $\times 2$ test and MannWhitney $U$ test for the constant variables. We made the assessments with SPSS ver. 11.0. This study considered the $p$ values lower than 0.05 as significant.

\section{Results:}

From 2014 to 2019, we operated 40 patients with renal tumor, atrophic kidney and non-functional kidney in our clinic and hospital during the study. 30 patients met the inclusion criteria and attended the study. We randomly included 15 patients into the laparoscopic nephrectomy (LN) group and 15 patients into the open nephrectomy $(\mathrm{ON})$ group.

Table-I : Demographic characteristics and perioperative data.

\begin{tabular}{lccc}
\hline & Laparoscopy $\mathrm{n}=15$ & Open $\mathrm{n}=15$ & P value \\
\hline Age (yr ) & $48 \pm 5$ & $46 \pm 7$ & 0.854 \\
BMI & $27.9 \pm 3.6$ & $29 \pm 4.5$ & 0.750 \\
Male/ Female & $8 / 7$ & $8 / 7$ & 0.810 \\
Duration of surgery (min ) & $114 \pm 29$ & $120 \pm 42$ & 0.508 \\
Indication & Atropic kidney : & Nonfunctional kidney :10 & \\
& 12Tumour :3 & Tumour :5 & 0.860 \\
PACU (0-4 Hrs)Analgesic use (n) & 3 & 2 & 1 \\
Early (5-9 Hrs in Ward Analgesic use (n) & 5 & 5 & \\
\hline
\end{tabular}

Values are mean $\pm s d P>0.05 n+$ number of patients or events, PACU : Post anesthesia care unit 
Table 1 outlines the basic characteristics of the study; we compared the two groups in terms of demographic characteristics and perioperative data. Average operation duration for the $\mathrm{LN}$ and $\mathrm{ON}$ groups were $114(60-170)$ and $120(70-180)$ respectively $(\mathrm{p}=0.508)$. None of the groups developed intra-operative complications and required transition to laparotomy. The study found no significant difference in postoperative complications.

Table-II : Post-operative pain scores regarding VAS assessment.

\begin{tabular}{lccc}
\hline VAS & Laparoscopy & Open & P \\
\hline 0 hour & $5.95 \pm 2.00$ & $5.71 \pm 1.80$ & 0.87 \\
2 hour & $3.79 \pm 1.57$ & $4.59 \pm 1.45$ & 0.76 \\
4 hour & $3.19 \pm 1.27$ & $3 \pm 1.18$ & 0.88 \\
8 hour & $2.47 \pm 1.14$ & $2.65 \pm 1.31$ & 0.89 \\
12 hour & $2.16 \pm 1.06$ & $2.45 \pm 1.18$ & 0.79 \\
18 hour & $1.56 \pm 1.08$ & $1.76 \pm 0.83$ & 0.88 \\
24 hour & $1.04 \pm 0.61$ & $1.31 \pm 0.89$ & 0.86 \\
\hline
\end{tabular}

Values are mean $\pm \mathrm{Sd} P>0,05$, Visual analogue scale (VAS) score

Table 3 : Series 1 Laparoscopy, Series 2 : Open, Visual analogue scale (VAS) score, $\mathrm{P}>0,05$.

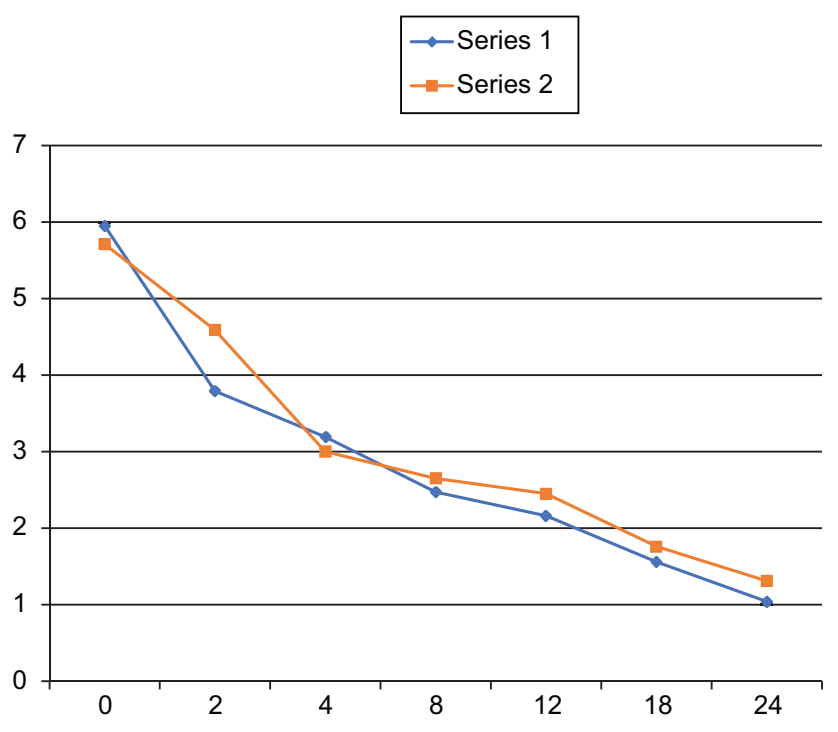

Table 2 and Table 3 demonstrate the post-operative pain scores regarding VAS assessment. Comparing the patients in the LN group to those in the ON group, the former had significantly less pain and displayed a significant reduction with respect to analgesic requirement.

\section{Discussion:}

As the less invasive endoscopic, percutaneous and laparoscopic methods have gradually developed and become widespread, open surgery, which is the most important element of urological practices, has started to be used less frequently. One of the main reasons for this change lies in the more invasive nature of the classical surgery than the modern surgical methods. However, due to the necessary equipments for the modern methods and the difficulties in gaining experience in these approaches, classical surgery remains the only alternative for some significant indications, and it holds its indispensable position in urology owing to its advantages in terms of costs and such reasons as specialist physician training and patient preferences in spite of being preferred less frequently in these days. Subcostal flank incision is generally employed in open surgery approaches for upper urinary system. This approach offers a wide operative field. However, according to the general consensus, it requires a considerable amount of muscle cutting, and leads to more postoperative pain and longer recovery period. Since laparoscopic nephrectomy was defined that it has been increasingly used in the field of urological laparoscopic surgery.[4] Especially, most significant advantages of the retroperitoneal approach, which was popularized in the last 10 years, can be named as early control of the renal artery, maintenance of peritoneal integrity, and lesser dissection requirement (e.g. column deflection) defined the retroperitoneal approach in $1993 .{ }^{5}$ Thanks to this method, it is possible to access the renal artery and vein in a shorter time frame. ${ }^{6}$ Orientation and narrow operative field are its most significant disadvantages. This approach shall be preferred in patients with a history of prior abdominal surgery. Reported to produce very successful results, this technique has some advantages over the standard laparoscopy such as enabling quicker vascular control, shorter operation duration, and earlier oral intake tolerance by the patients. ${ }^{5-9}$ LRN has become the standard surgical approach in many centers for the surgical treatment of renal tumors. Beyond the discussion over the preference of transperitoneal approach or retroperitoneal approach, current observations demonstrate the perioperative advantages of laparoscopic approach over open surgery approach. In a multi-centered study, investigators have compared the results of 103 LRN (85 transperitoneal; 18 retroperitoneal) operation with 
46 classical open radical nephrectomy operation.[10] They have reported an average blood loss figure of $254 \mathrm{ml}$ vs. $465 \mathrm{ml}$ and a transfusion figure of $5 \%$ vs. $9 \%$ respectively. In a similar comparative study, the investigators have reported a blood loss figure of 97 $\mathrm{ml}$ vs. $295 \mathrm{ml}$, and a complication rate of $13 \%$ vs. $24 \%$. An assessment of the data found in the literature demonstrates a minor complication rate of $3 \%-15 \%$, and a major complication rate of $3 \%-8 \% .{ }^{11}$ The complication rates in open surgery approaches employed at similar clinical phases have been reported to be between $10 \%$ and $20 \%$.[12] While the average operation duration was reported to be 240 minutes in the initial period, it has reduced to an average of 150 minutes along with the enhanced experience in this approach. Up until today, studies have reported a significant advantage in favor of LRN in terms of hospital stay, the investigators have reported $1,4 \mathrm{vs}$. 5,8 days whereas others have reported 4,8 vs. 9,7 days. ${ }^{9}, 12$ Laparoscopic practices have recently become popular due to such benefits as quick mobilization and early discharge, low rate of late and early postoperative complication, and early postoperative recovery. ${ }^{12-16}$ Despite their potential of temporary intra-operative cardiovascular and respiratory dysfunctions, even the old patients with accompanying diseases are more frequently planned for such practices. ${ }^{17}$ Our study and other prior studies have found that some post-operative patients feel constant pain. ${ }^{18,19}$ These are more similar to the incidences prolonging the return to normal activity and resulting from severe pain such as respiratory and hemodynamic changes. In this study, we observed same level of reduction in pain and feeling good at the 24th hour in both groups (Tables II-III). A survey has been developed based on the thesis that the painful stimulant is objectively countable and predictable, and proportional subjective pain scale is the only viable and effective method to assess pain. ${ }^{20}$ And this study employed this method. When pain is more characterized, it has an experimental experience notably associated with multifactorial physical origin and the peripheral and central neural feedback mechanism and inhibition of senses. ${ }^{21,22}$ Pain score might be associated with a patient's preoperative preparation, pain sensitivity and personal expectations. ${ }^{22}$ As all the patients included in this study were prepared in a similar way, preoperative patient preparation and the differences in their sensitivity do not lead to the changes in our findings. Demographic differences might lead to the distribution of pain expectation and - theoretically - might explain some results of this study. ${ }^{21}$ Since the data obtained are similar, this should be ignored for the patients included in this study. According to the findings of this study, pain scores are similar in both groups, but the pethidine consumption is lesser in the LN group than the ON group. Between 1998 and 2006, some investigators observed retrospectively compared the results of LRN (41 patients) and ORN (71 patients) operations on T2 tumors. ${ }^{23}$ They did not randomize the patients, and performed laparoscopy by employing transperitoneal or retroperitoneal approach. The findings of this study demonstrated the advantages of LRN over ORN in terms of blood loss ( $246 \mathrm{ml}$ vs. $537 \mathrm{ml})$, transfusion rate $(15 \%$ vs. $32 \%)$ requirement for morphine-like analgesic (16 mg vs. $35 \mathrm{mg}$ ), hospital stay (3,6 days vs. 6,6 days), and recovery period (1,6 weeks vs. 3,3 weeks). Postoperative complication rates were similar (12\% vs. $15 \%)$. In an average follow-up period of 51 and 57 months, non-recurrent $(90 \%$ vs. $92 \%)$, cancerspecific ( $94 \%$ vs. $95 \%)$, and general ( $89 \%$ vs. $88 \%$ ) survival rates demonstrated no statistical difference. Both groups had an average tumor size of $10 \mathrm{~cm}$ approximately. Authors acknowledge the short-term advantages of laparoscopic approach. However, there is only one randomized controlled study comparing LRN and ORN designed on the hypothesis that such big-sized tumors are challenging and only experienced surgeons should make an intervention on such tumors ${ }^{23}$. Although surgical intervention was performed for both benign and malign diseases and the maximum tumor size was $8 \mathrm{~cm}$, the groups formed among 45 patients were well-matched. There was no significant difference between the groups with regard to operation duration (105 minutes vs. 93 minutes) or unexpected hospital stay (4 days vs. 5 days). Not surprisingly, postoperative pain was less in the LRN group (3,6 vs. 5,4 on a 10-point visual analogue scale), but there was no difference in the third month. Quick return to normal activities was again in favor of the laparoscopic group (42 days vs. 62 days).

Study limitations: A questionnaire used to evaluate the pain. In the future more than one questionnaires can be used for the same groups. On the other hand groups have small sample size and they can have more samples.

\section{Conclusion:}

As a result, laparoscopic applications compared to open surgery; early mobilization, early and late postoperative complication rate is low and is more advantageous in terms of short-term hospitalization. However, in the early postoperative recovery period 
pethidine requirement is more in open surgery, but shows similar characteristics in terms of pain scores.

\section{References:}

1. Bonica, JJ. The need of a taxonomy. Common wealth of Australia, National Health and Medical Research Council, acute Pain. Pain 1979; 6:247

2. Weinbroum, AA . A single small dose of postoperative ketamine provides rapid and sustained improvement in morphine analgesia in the presence of morphine-resistant pain. Anesth Analg 2003;96: 789 -795.

3. Eypasch E, Sauerland S, Lefering R, Neugebauer EA .Laparoscopic versus open appendectomy: between evidence and common sense. Dig Surg2002; 19: 518-522.

4. Clayman RV, Kavoussi LR, Soper NJ, Dierks SM, Meretyk S, et al. aparoscopic nephrectomy: initial case report. J Urol 1991;146: 278-282.

5. Gaur DD, Agarwal DK, Purohit KC . Retroperitoneal laparoscopic nephrectomy: initial case report. J Urol 1993;149: 103-105.

6. Batler RA, Campbell SC, Funk JT, Gonzalez CM, Nadler RB . Hand-assisted vs. retroperitoneal laparoscopic nephrectomy.J Endourol 2001;15: 899-902.

7. Desai MM, Strzempkowski B, Matin SF. Prospective randomized comparison of transperitoneal versus retroperitoneal laparoscopic radical nephrectomy. J Urol 2005; 173:38-41.

8. Cicco A, Salomon L, Hoznek A, Saint F, Alame $\mathrm{W}$, et al. Results of retroperitoneal laparoscopic radical nephrectomy. J Endourol 2001;15:355-359.

9. Abbou CC, Cicco A, Gasman D . Retroperitoneal laparoscopic versus open radical nephrectomy. J Urol 1999; 161:1776-1780.

10. Ono Y, Kinukawa T, Hattori R, Gotoh M, Kamihira $\mathrm{O}$, et al. The long-term outcome of laparoscopic radical nephrectomy for small renal cell carcinoma. J Urol 2001;165: 1867-1870.

11. Gill IS, Schweizer D, Hobart MG, Sung GT, Klein EA . Retroperitoneal laparoscopic radical nephrectomy: The Clevland Clinic experience. J Urol 2000;163: 1665-1670.
12. Swanson DA, Borges PM . Complications of transabdominal radical nephrectomy for renal cell carcinoma. J Urol 1983;129: 704-707.

13. Eypasch E, Sauerland S, Lefering R, Neugebauer EA .Laparoscopic versus open appendectomy: between evidence and common sense. Dig Surg 2002;19: 518-522.

14. Weinbroum AA . A single small dose of postoperative ketamine provides rapid and sustained improvement in morphine analgesia in the presence of morphine-resistant pain. Anesth Analg2003; 96: 789-795.

15. Kapfer B, Alfonsi P, Guignard B, Sessler DI, Chauvin M. Nefopam and ketamine comparably enhance postoperative analgesia. Anesth Analg2005; 100: 169-174.

16. Weinbroum AA, Lalayev G, Yashar T, BenAbraham R, Niv D, et al. Combined pre-incisional oral dextromethorphan and epidural lidocaine for postoperative pain reduction and morphine sparing: a randomised double-blind study on daysurgery patients. Anaesthesia2001; 56: 616622.

17. Andersson LE, Baath M, Thorne A . Effect of carbon dioxide pneumoperitoneum on development of atelectasis during anesthesia. Anesthesiology2005; 102: 293-299.

18. Kapfer B, Alfonsi P, Guignard B, Sessler DI, Chauvin M. Nefopam and ketamine comparably enhance postoperative analgesia. Anesth Analg 2005; 100: 169-174.

19. Haughton VM, Fine J .Measuring the effect of novel therapies for back pain. AJNR Am J Neuroradiol2003; 24: 784-787.

20. Katz J, Melzack R. Measurement of pain. Surg Clin North Am 1999; 79: 231-252.

21. Sorkin LS . Basic pharmacology and physiology of acute pain processing. Anesthesiol Clin North Am1997; 15: 235-249.

22. Caumo W, Schmidt AP, Schneider CN .Preoperative predictors of moderate to intense acute postoperative pain in patients undergoing abdominal surgery. Acta Anaesthesiol Scand2002; 46: 1265-1271.

23. Holdcroft A, Power I . Recent developments: management of pain. BMJ 2003;326: 635-639. 